\title{
Conexões
}

Educação Física, Esporte e Saúde

\section{Níveis de ansiedade de atletas no início e no final de um campeonato de basquetebol}

\author{
Bruna Alves Santana ${ }^{1}$ \\ Higor dos Santos de Souza ${ }^{1}$ \\ Bruna Maria Genuíno Sousa ${ }^{1}$ \\ Douglas Versuti Arantes Alvarenga ${ }^{1}$ \\ Rosana Lopes da Silva Garcia ${ }^{2}$ \\ Ivan Wallan Tertuliano ${ }^{3}$
}

\section{RESUMO}

Objetivo: O objetivo desse estudo foi investigar o nível de ansiedade pré-competitiva de atletas de basquetebol, das categorias de base de um clube esportivo, comparando o nível de ansiedade pré-competitiva no início e no final do campeonato de 2018. Metodologia: Para isso, participaram do estudo 26 atletas, voluntários, do sexo masculino, federados, com idades entre 13 e 15 anos. Os atletas responderam o Competitive State Anxiety Inventory - 2r (CSAI-2r). Resultados e discussão: Os resultados demonstraram que os atletas apresentaram escores elevados de autoconfiança e valores baixos de ansiedade somática, tanto no início, quanto no final do campeonato de 2018. Além disso, durante o campeonato, os valores de autoconfiança mantiveram-se estáveis, sendo sempre superiores aos valores de ansiedade. Conclusão: Pode-se concluir que durante o campeonato de 2018 os atletas apresentaram um elevado escore de autoconfiança e que este fator tem influência direta nos valores baixos de ansiedade cognitiva e somática, corroborando a teoria Multidimensional da ansiedade.

Palavras-chave: Ansiedade. Basquetebol. Psicologia do esporte. CSAI-2R.

${ }^{1}$ Centro Universitário Adventista de São Paulo, São Paulo - SP, Brasil.

2 Centro Universitário Ítalo Brasileiro, São Paulo - SP, Brasil.

3 Universidade Anhembi Morumbi, São Paulo - SP, Brasil.

\section{Correspondência:}

Ivan Wallan Tertuliano. Universidade Anhembi Morumbi, Escola de Ciências da Saúde, Educação Física, Rua Doutor Almeida Lima, 1134, Mooca, CEP 03164000, São Paulo, SP - Brasil, E-mail: ivanwallan@gmail.com 


\section{Athlete anxiety levels at beginning and end of a basketball championship}

\section{ABSTRACT}

Objective: The aim of this study was to investigate the pre-competitive anxiety level of basketball athletes from the base categories of a sports club by comparing the precompetitive anxiety level at the beginning and end of the 2018 championship. Methodology: For this, 26 male athletes, volunteers, federated, aged between 13 and 15 years participated in the study. The athletes responded the Competitive State Anxiety Inventory - $2 r(C S A I-2 r)$. Results and discussion: The results showed that the athletes had high self-confidence scores and low somatic anxiety values, both at the beginning and at the end of the 2018 championship. In addition, during the championship, self-confidence values remained stable, always higher than anxiety values. Conclusion: It can be concluded that during the 2018 championship the athletes presented a high self-confidence score and that this high score has a direct influence on the low values of cognitive and somatic anxiety, corroborating the Multidimensional Anxiety Theory.

Keywords: Anxiety. Basketball. Sport psychology. CSAI-2R.

\section{Niveles de ansiedad del atleta al comienzo y al final de un campeonato de baloncesto}

\section{RESUMEN}

Objetivo: El objetivo de este estudio fue investigar el nivel de ansiedad precompetitivo de los atletas de baloncesto de las categorías básicas de un club deportivo mediante la comparación del nivel de ansiedad precompetitiva al comienzo y al final del campeonato 2018. Metodología: 26 atletas voluntarios varones, de 13 a 15 años, participaron en el estudio. Los atletas respondieron el Inventario de ansiedad de estado competitivo - $2 r$ (CSAI-2r). Resultados y discusión: Los resultados mostraron que los atletas tenían altos puntajes de autoconfianza y bajos valores de ansiedad somática, tanto al comienzo como al final del campeonato 2018. Además, durante el campeonato, los valores de autoconfianza se mantuvieron estables, siempre más altos que los valores de ansiedad. Conclusión: Se puede concluir que durante el campeonato 2018 los atletas tuvieron un alto puntaje de autoconfianza y que este alto puntaje tiene una influencia directa en los bajos valores de ansiedad cognitiva y somática, corroborando la Teoría de la ansiedad Multidimensional.

Palabras Clave: Ansiedad. Baloncesto. Psicología del deporte. CSAI-2R. 


\section{INTRODUÇÃO}

No ambiente do esporte competitivo a ansiedade é compreendida como um dos fatores que influenciam diretamente o desempenho dos atletas (WEINBERG; GOULD, 2017), sendo essa influência, muitas vezes, negativa para o desempenho (MACHADO, 2006). Isso ocorre pois a ansiedade é uma resposta emocional de aversão ao estresse, que resulta de uma avaliação de ameaça, sendo caracterizada por sentimentos subjetivos de incapacidade para realizar a tarefa (INTERDONATO et al., 2010).

A ansiedade pode ser caracterizada como ansiedade traço e ansiedade estado (WEINBERG; GOULD, 2017). Ansiedade estado refere-se a diferenças individuais relativamente estáveis que levam à percepção de situações ameaçadoras, de acordo com as experiências pessoais adquiridas (SPIELBERGER; GORSUCH; LUSHENE, 1970; MACHADO, 2006). Em contrapartida, a ansiedade de estado refere-se a sinais momentâneos referentes a percepções de situações ameaçadoras (SPIELBERGER, 1972; PALUDO et al., 2016).

Diante do apresentado, alguns instrumentos foram desenvolvidos com o intuito de avaliar a influência da ansiedade no rendimento esportivo. Todavia, o primeiro instrumento desenvolvido para avaliar ansiedade não tinha como objetivo o contexto esportivo, mas foi utilizado, também, no ambiente esportivo. Este instrumento, o STAI (State-Trait Anxiety Inventory), foi desenvolvido por Spielberger, Gorsuch e Lushene (1970) e no Brasil recebeu o nome de IDATE (Inventário de Ansiedade Traço-Estado) (BIAGGIO; NATALÍCIO; SPIELBERGER, 1977). A partir deste, outros instrumentos foram desenvolvidos, buscando compreender melhor os fatores estressores e as reações dos atletas ansiosos, ampliando o entendimento sobre a influência da ansiedade no rendimento esportivo.

Dentre esses outros instrumentos, cita-se o CSAI (Competitive State Anxiety Inventory) (MARTENS et al., 1980), desenvolvido com o intuito de mensurar a ansiedade estado em atletas, assumindo a multidimensionalidade da ansiedade. Após uma revisão de conteúdo e constructo, o CSAI recebeu uma segunda versão, o CSAI-2 (Competitive State Anxiety Inventory-2), proposta por (MARTENS et al., 1990) O CSAI-2 tendo a função de mensurar a ansiedade estado competitiva a partir de três subescalas: ansiedade cognitiva, ansiedade somática e autoconfiança. Esse instrumento é composto por 27 questões com respostas do tipo Likert. Anos depois, o CSAI-2 passou por nova validação de constructo e uma versão reduzida (CSAI-2r) foi apresentada (COX; MARTENS; RUSSELL, 2003), contendo 17 questões.

O CSAI-2 foi validado para o Brasil (COELHO; VASCONCELOS-RAPOSO; MAHL, 2010), além de apresentar uma versão reduzida, o CSAI-2r (COX; MARTENS; RUSSELL, 2003), que também foi validada e traduzida para o Brasil 
(FERNANDES; VASCONCELOS-RAPOSO; FERNANDES, 2012a). O CSAI-2r contém menos itens que o original (17 itens), porém com a mesma qualidade psicométrica (FERNANDES et al., 2014; PALUDO et al., 2016). Além disso, esses instrumentos (CSAI-2 e CSAI-2r) foram utilizados em algumas pesquisas e demonstraram bons índices de confiabilidade (COELHO; VASCONCELOS-RAPOSO; MAHL, 2010; COX; MARTENS; RUSSELL, 2003; FERNANDES et al., 2014; FORTES et al., 2017), apontando a viabilidade dos mesmos para mensuração dos níveis de ansiedade pré-competitiva.

Utilizando-se do CSAI-2 e/ou CSAI-2r, alguns estudos foram realizados (ARRUDA et al., 2017; FERNANDES; VASCONCELOS-RAPOSO; FERNANDES, 2012a; 2012b; HAGAN; POLLMANN; SCHACK, 2017; MILAVIĆ; JURKO; GRGANTOV, 2013; NASCIMENTO JUNIOR et al., 2016; PAES et al., 2016; PALUDO et al., 2016; PONS et al., 2018; TEIXEIRA; NUNES; CRUZ, 2016; TERTULIANO et al., 2019) e o conjunto de resultados apresentam-se inconclusivos, pois, de acordo com Paludo et al. (2016), apenas parte dos estudos apresentam relação entre ansiedade e desempenho, demonstrando a necessidade de estudos comprobatórios sobre a relação entre tais fatores.

Além disso, os estudos foram conduzidos com esportes individuais (BARRETO, 2017; COELHO; VASCONCELOS-RAPOSO; MAHL, 2010; FERNANDES; VASCONCELOS-RAPOSO; FERNANDES, 2012a; FORTES et al., 2017; TEIXEIRA; NUNES; CRUZ, 2016; TERTULIANO et al., 2019) ou esportes coletivos variados (ARRUDA et al., 2017; BERNARDT; SEHNEM, 2017; HAGAN; POLLMANN; SCHACK, 2017; NASCIMENTO JUNIOR et al., 2016; PAES et al., 2016; PONS et al., 2018; SONOO et al., 2010), tendo apenas 1 dos estudos conduzido, exclusivamente, com atletas de basquetebol (ARRUDA et al., 2017), justificando-se o presente estudo.

Tratando do basquetebol, é oportuno citar que a regra dos 24 segundos para conclusão do ataque é um fator que pode influenciar os níveis de ansiedade dos atletas, justificando estudos com a modalidade, pois esse fator temporal, para conclusão de uma jogada, é exclusivo do basquetebol. Tal variável (o tempo de 24 segundos para finalização) pode contribuir com o aumento dos níveis de ansiedade, principalmente se a equipe necessita da vitória para continuar na competição, ou para conquistar o título, em uma final. Assim, o estudo com atletas de basquetebol se torna relevante.

Contribuindo com isso, o estudo de Arruda et al. (2017), que investigou atletas de basquetebol, apresenta a limitação de utilizar-se de atletas de elite, demonstrando a necessidade de estudos com as categorias de base do basquetebol, justificando-se, novamente, o presente estudo. Além disso, os estudos supracitados foram conduzidos com a análise de apenas um momento da competição, o que demonstra a não percepção de que a ansiedade de estado pode modificar-se durante um campeonato (WEINBERG; GOULD, 2017). 
Por fim, ao assumir o constructo multidimensional da ansiedade, é esperado que os atletas, ao apresentaram elevados níveis de ansiedade (cognitiva e somática), apresentem baixos níveis de autoconfiança (FERNANDES; VASCONCELOS-RAPOSO; FERNANDES, 2012a; FORTES et al., 2017; PONS et al., 2018; TERTULIANO et al., 2019). Portanto, o objetivo desse estudo foi investigar o nível de ansiedade pré-competitiva de atletas de basquetebol, das categorias de base de um clube esportivo de São Paulo, comparando o nível de ansiedade précompetitiva no início e no final do campeonato.

\section{MÉTODO}

\section{Participantes}

A amostra foi composta por 26 atletas, das categorias de base, de um clube esportivo da cidade de São Paulo. Foram usados os seguintes critérios de inclusão: sexo masculino, federados, com idades entre 13 e 15 anos $(M=14,19, D P=0,63)$, atletas de basquetebol e que aceitassem participar voluntariamente da pesquisa. Todos os atletas foram comandados, na temporada avaliada, pela mesma equipe técnica (preparador físico, técnico e psicólogo esportivo) e que eram pertencentes a duas categorias do basquetebol, a sub-13 (12 participantes) e a sub-15 (14 participantes).

Além disso, todos os atletas disputaram o Campeonato Paulista de basquetebol da Grande São Paulo, organizado pela Federação Paulista de basquetebol, evento que foi utilizado para avaliação. Enquanto tempo de equipe, os participantes apresentaram uma média de 30,1 meses ( $D P=27,8$ meses). Em relação as posições dos jogadores, 6 atletas atuam como Ala, 7 como Ala/Pivô, 5 como Amador, 1 como Lateral e 7 como Pivô.

\section{INSTRUMENTOS}

Foram utilizados dois instrumentos: a) Questionário de Caracterização Sociodemográfica e b) CSAI-2r, proposto por Cox, Martens e Russell (2003) e validado para o Brasil (FERNANDES; VASCONCELOS-RAPOSO; FERNANDES, 2012a). Cabe citar que assumindo-se a qualidade psicométrica do instrumento, optou-se pela utilização da versão reduzida do CSAI-2, a qual apresenta as mesmas qualidades psicométricas que a versão ampliada, mas com a qualidade de ser mais rápida a aplicação, em função de apresentar menos itens (FERNANDES et al., 2014; PALUDO et al., 2016; TERTULIANO et al., 2019).

O questionário de caracterização foi elaborado especificamente para o estudo, com questões relacionadas a perfil pessoal, posição que atua e esporte que pratica. O CSAI-2r é um instrumento composto por 17 itens, que avaliam três subescalas: ansiedade cognitiva (itens 2, 5, 8, 11 e 14), ansiedade somática (itens 
$1,4,6,9,12,15$ e 17) e autoconfiança (itens 3, 7, 10, 13 e 16). Além disso, essas subescalas podem ser avaliadas de acordo com as dimensões: intensidade, frequência e direção (COX; MARTENS; RUSSELL, 2003). No presente estudo optou-se por avaliar somente a dimensão intensidade, pois a literatura aponta que é possível compreender os níveis de ansiedade pré-competitiva com o uso apenas dessa dimensão (FERNANDES; VASCONCELOS-RAPOSO; FERNANDES, 2012a; TERTULIANO et al., 2019).

As questões foram preenchidas de acordo com uma escala do tipo Likert de quatro pontos, alternando de 1 (nada) a 4 (muito). Assim, calculando o escore de cada subescala deve-se somar as respostas dos itens de cada e dividir pelo respectivo número de itens, podendo variar entre 1 e 4 o valor da subescala.

\section{Procedimentos}

A presente pesquisa obteve aprovação do Comitê de Ética e Pesquisa (CEP) do Hospital e Centro de Reabilitação da AACD e aprovado pelo mesmo comitê, sob o número de parecer: 1.541.273. Por se tratar de um estudo com seres humanos, a presente pesquisa respeitou os princípios legais que envolve coleta de dados com os mesmos, respeitando os padrões éticos e metodológicos de pesquisas com seres humanos do Conselho Nacional de Saúde (CNS), resolução 466/12.

Após a devida autorização do CEP, realizou-se contato prévio com os responsáveis do clube, com o intuito de explanar o objetivo da pesquisa e solicitar autorização para a coleta. Na sequência, realizou-se contato com os participantes para informar os propósitos da pesquisa. Assim, os atletas foram informados dos objetivos do estudo. A próxima etapa consistiu na assinatura do Termo de Consentimento Livre e Esclarecido (TCLE), garantindo-se, dessa forma, o anonimato de todas as informações coletadas. No presente estudo, os participantes são todos menores de 18 anos. Diante disso, o TCLE foi assinado por um responsável, maior de idade, e o menor assinou o termo de assentimento. Dessa forma, todos os participantes preencheram e assinaram o termo de assentimento e seus responsáveis o Termo de Consentimento Livre e Esclarecido (TCLE).

O Questionário de Caracterização Sociodemográfica foi respondido apenas uma vez, no início da competição de 2018 e o CSAI-2r foi respondido em dois momentos: no início da competição de 2018 (Abril), junto com o questionário supracitado, no dia da primeira partida do campeonato (entre 2 horas e 1 hora antes da partida) e no final da competição de 2018 (Novembro), no dia do jogo final do campeonato, o que coincidiu, para as duas equipes, com as finais da competição (sub-13 e sub-15), sendo respondido entre 2 horas e 1 hora antes da partida. Dessa maneira, o presente estudo é caracterizado como um estudo longitudinal, pois acompanhou os atletas ao longo de uma competição. 
Para garantir a confiabilidade das informações coletadas, os questionários foram respondidos individualmente, na presença de ao menos um dos pesquisadores. Dessa forma, não houve troca de informações entre os atletas, antes e durante a aplicação dos questionários. O tempo de preenchimento dos questionários foi de aproximadamente 20 minutos. Após todos atletas responderem os questionários, as informações coletadas foram transferidas para uma planilha eletrônica (Excel, versão 2016) para análise dos resultados.

\section{ANÁLISE EstATÍSTICA}

Em função do número de participantes, houve a necessidade de testar a normalidade e a homogeneidade de variância dos dados, o que foi realizado por meio dos seguintes testes: Kolmogorov-Smirnov (K-S) para normalidade e o teste de Levene para homogeneidade de variância. Esses procedimentos foram adotados no questionário CSAI-2r respondido no início e no final da competição, separadamente. Neste estudo as análises inferenciais assumiram o valor de $\mathrm{p} \leq$ 0,05 para níveis de significância. Como os dados não apresentaram distribuição normal $(p<0,05)$, nem homogeneidade de variância $(p<0,05)$, para ambos os momentos de coleta, foram utilizados: Mediana (Md) e Quartis (Q1; Q3) para caracterização dos resultados e para as análises assumiu-se testes não paramétricos.

Com o intuito de avaliar a confiabilidade das subescalas do instrumento, ou seja, avaliar a consistência interna das subescalas do questionário, em cada momento de coleta, utilizou-se do alfa de Cronbach (a de Cronbach), assumindo valores entre 0,7 e 0,8 como aceitáveis para o a (FIELD, 2009), para cada subescala e momento de coleta, de forma isolada. Para avaliação da fidedignidade teste-reteste foi utilizado o coeficiente de correlação intraclasses entre os itens e subescalas do instrumento.

Os valores de correlação foram avaliados conforme proposto por Hopkins (2002), em que <0,10 (trivial), 0,10 a 0,30 (baixa), 0,31 a 0,50 (moderada), 0,51 a 0,70 (alta), 0,71 a 0,90 (muito alta), 0,91 a 0,99 (quase perfeita) e 1 (perfeita). No presente estudo assumiu-se valores de correlação acima de 0,31 (moderado), em função do número de participantes (FIELD, 2009). Após essas etapas de análises, iniciou-se as análises acerca do objetivo do estudo. Para analisar as diferenças entre as subescalas, em cada momento de coleta, utilizou-se o teste de Friedman.

Concernente à localização, utilizou-se o post hoc de Wilcoxon. Para controle do erro tipo 1, foi utilizado o procedimento sequencial Holm de Bonferroni (GREEN; SALKIND; AKEY, 2000). Para as análises entre os momentos de coleta (início $X$ final da competição), utilizou-se o teste de Wilcoxon, comparando-se os resultados do início e do final da competição. Todas as análises foram realizadas com o auxílio do IBM SPSS Statistics, versão 22. 


\section{REsultados E DiscussÃo}

Em relação à confiabilidade das escalas, o alpha de Cronbach revelou bons índices de confiabilidade no início $(a>0,73)$ e no final da competição $(a>0,75)$, ou seja, o índice de consistência interna geral do instrumento foi satisfatório nos dois momentos de coleta. Porém, como o instrumento é multidimensional, houve a necessidade de calcular a confiabilidade de cada subescala, em cada momento de coleta, isoladamente. De acordo com a tabela 1 , os valores de confiabilidade para cada subescala, no início da competição, variaram entre 0,73 e 0,86, tendo a correlação item-subescala variando entre 0,36 e 0,76, indicando correlação adequada entre os itens e a subescala a qual ele pertence, pois variou entre moderada (itens 1, 5, 8, 12 e 15), alta (itens 2, 3, 4, 6, 9, 10, 11, 13, 14, 16 e 17) e muito alta (item 7).

Tabela 1 - Consistência interna das subescalas do CSAI-2r e correlação itemsubescala $(n=26)$, início da competição

\begin{tabular}{|c|c|c|c|}
\hline Subescala & Item número & $a$ & Correlação Item-Subescala \\
\hline Ansiedade Cognitiva & $2,5,8,11$ e 14 & 0,784 & $\begin{array}{c}0,637 / 0,402 / 0,454 / 0,706 / \\
0,613\end{array}$ \\
\hline Ansiedade Somática & $\begin{array}{c}1,4,6,9,12,15 \mathrm{e} \\
17\end{array}$ & 0,727 & $\begin{array}{c}0,363 / 0,525 / 0,514 / 0,512 / \\
0,426 / 0,378 / 0,685\end{array}$ \\
\hline Autoconfiança & $3,7,10,13$ e 16 & 0,864 & $\begin{array}{c}0,707 / 0,760 / 0,595 / 0,674 / \\
0,700\end{array}$ \\
\hline
\end{tabular}

Dando continuidade aos dados de confiabilidade, de acordo com a tabela 2, os valores de confiabilidade para cada subescala, no final da competição, variaram entre 0,75 e 0,80, tendo a correlação item-subescala variando entre 0,35 e 0,73, indicando correlação adequada entre os itens e a subescala que ele pertence, pois houve variação moderada (itens $1,2,3,9,14$ e 15), alta $(4,5,6,7,8,10,12,13$ e 17) e muito alta (11 e 16) entre item-subescala.

Tabela 2 - Consistência interna das subescalas do CSAI-2r e correlação itemsubescala $(n=26)$, final da competição

\begin{tabular}{|c|c|c|c|}
\hline Subescala & Item número & $\mathbf{a}$ & Correlação Item-Subescala \\
\hline Ansiedade Cognitiva & $2,5,8,11$ e 14 & 0,797 & $\begin{array}{c}0,452 / 0,613 / 0,623 / 0,728 / \\
0,485\end{array}$ \\
\hline Ansiedade Somática & $\begin{array}{c}1,4,6,9,12,15 \\
\text { e } 17\end{array}$ & 0,799 & $\begin{array}{c}0,355 / 0,565 / 0,564 / 0,492 / 0,624 / \\
0,497 / 0,615\end{array}$ \\
\hline Autoconfiança & $3,7,10,13$ e 16 & 0,752 & $\begin{array}{c}0,428 / 0,700 / 0,703 / 0,511 / \\
0,717\end{array}$ \\
\hline
\end{tabular}

Frente as análises para cada momento de coleta, para as 3 subescalas do instrumento, observa-se pela análise descritiva (Tabela 3), realizada com os dados do início da competição, que o maior escore foi observado na subescala autoconfiança $(M d=3,20)$, e o mais baixo na subescala ansiedade somática $(M d=1,57)$. Tais informações foram suportadas pela análise inferencial, pois o 
teste de Friedman apresentou diferença significante entre as subescalas $\left[\mathrm{x}^{2}(2)=\right.$ 29,961; $p<0,0001]$.

Concernente à localização, o teste de Wilcoxon demonstrou escores superiores para subescala autoconfiança ( $p<0,016$, valor ajustado), em relação às demais subescalas e, além disso, apresentou escores menores para subescala ansiedade somática ( $p<0,016$, valor ajustado), em relação às demais subescalas. Tais resultados demonstraram que no início da competição os atletas apresentaram-se autoconfiantes e com baixa ansiedade somática.

Tabela 3 - Comparação das subescalas do CSAI-2r para toda amostra $(n=26)$, início da competição

\begin{tabular}{cc}
\hline Subescala & Itens \\
\hline Ansiedade Cognitiva & $2,00(1,75 ; 2,40)$ \\
Ansiedade Somática & $1,57(1,29 ; 2,07)$ \\
Autoconfiança & $3,20(2,80 ; 4,00)$ \\
\hline
\end{tabular}

Analisando-se os dados do final da competição, observa-se pela análise descritiva (Tabela 4), que os valores são muito similares aos valores do início da competição, tendo sido observado que a subescala autoconfiança apresentou os maiores escores $(M d=3,40)$, e a subescala ansiedade somática os mais baixos $(\mathrm{Md}=1,65)$. Tais informações foram suportadas pela análise inferencial, pois o teste de Friedman apresentou diferença significante entre as subescalas $\left[\mathrm{x}^{2}(2)=\right.$ 31,000; $\mathrm{p}<0,0001]$.

Concernente à localização, o post hoc demonstrou comportamento estatístico semelhante ao início da temporada, ou seja, a subescala autoconfiança apresentou diferença significante em relação às demais subescalas ( $p<0,016$, valor ajustado), com valores superiores e a subescala ansiedade somática apresentou-se, também, significativamente diferente das demais subescalas $(p<0,016$, valor ajustado), com os menores valores. Tais resultados demonstraram que no final da competição, assim como no início da competição, a subescala que obteve maior escore foi a autoconfiança e que a subescala ansiedade somática obteve os menores escores.

Tabela 4 - Comparação das subescalas do CSAI-2r para toda amostra $(n=26)$, final da competição

\begin{tabular}{cc}
\hline Subescala & Itens \\
\hline Ansiedade Cognitiva & $2,20(1,80 ; 2,60)$ \\
Ansiedade Somática & $1,65(1,43 ; 2,32)$ \\
Autoconfiança & $3,40(3,00 ; 3,80)$ \\
\hline
\end{tabular}

Nas comparações entre os momentos de coleta, pode-se observar (Tabela 5) que não houve diferença significativa entre as subescalas nos dois momentos da coleta. Tais informações foram suportadas pelo teste de Wilcoxon, o qual não apresentou diferença significante entre os períodos, para nenhuma subescala 
$(p>0,05)$. Assim, os resultados reforçam o observado nas análises anteriores, ou seja, os atletas apresentaram os mesmos níveis de ansiedade pré-competitiva no início e no final da competição.

Tabela 5 - Comparação das subescalas do CSAI-2r entre o início e o final da competição $(n=26)$

\begin{tabular}{ccccc}
\hline Subescala & $\begin{array}{c}\text { Início da } \\
\text { Temporada } \\
\text { Md }(\mathbf{Q 1} ; \mathbf{Q 3})\end{array}$ & $\begin{array}{c}\text { Final da Temporada } \\
\text { Md }(\mathbf{Q 1} ; \mathbf{Q 3})\end{array}$ & $\mathbf{Z}$ & $\mathbf{P}$ \\
\hline Ansiedade Cognitiva & $2,00(1,75 ; 2,40)$ & $2,20(1,80 ; 2,60)$ & $-1,231$ & 0,218 \\
Ansiedade Somática & $1,57(1,29 ; 2,07)$ & $1,65(1,43 ; 2,32)$ & $-1,530$ & 0,126 \\
Autoconfiança & $3,20(2,80 ; 4,00)$ & $3,40(3,00 ; 3,80)$ & $-0,474$ & 0,636 \\
\hline
\end{tabular}

Em síntese, os resultados demonstraram que os atletas de basquetebol, do clube esportivo investigado, apresentaram valores superiores para subescala autoconfiança, além de apresentarem valores inferiores para subescala ansiedade somática, nos dois momentos da pesquisa. Por fim, os resultados demonstraram que durante a competição de 2018 , os atletas não tiveram mudanças significantes nos níveis de ansiedade pré-competitiva, no que se refere a dimensão avaliada, ou seja, a intensidade.

Tratando-se do instrumento CSAI-2r, os resultados demonstraram valores moderados de validade e fidedignidade, mas não fortes, corroborando parcialmente a literatura (FERNANDES; VASCONCELOS-RAPOSO; FERNANDES, 2012a; 2012b; PALUDO et al., 2016; TEIXEIRA, 2016; TERTULIANO et al., 2019). Entretanto, conforme aponta Field (2009), esses valores podem auxiliar na interpretação dos achados, haja vista o número baixo de participantes, limitando a extrapolação dos resultados, ou seja, limitando as explicações à população investigada. Retornando aos resultados, o presente estudo apresentou resultados de elevada autoconfiança e baixa ansiedade somática, independentemente de a análise ser do início ou do final da competição.

Esses resultados corroboram a hipótese da teoria Multidimensional da Ansiedade (MARTENS; VEALEY; BURTON, 1990), a qual diz que quando os atletas apresentam elevados níveis de autoconfiança, os escores de ansiedade somática e cognitiva se apresentam baixos. Dessa forma, a teoria cita que um dos elementos que pode regular a ansiedade é a autoconfiança, que tem relação com pensamentos positivos acerca do sucesso do atleta no esporte (WEINBERG; GOULD, 2017). Em outras palavras, a teoria aponta que existe uma correlação inversamente proporcional entre ansiedade e autoconfiança. Além disso, os resultados deste estudo corroboram os resultados apresentados pela literatura (BERNARDT; SEHNEM, 2017; FERNANDES et al., 2013; FERNANDES; VASCONCELOS-RAPOSO; FERNANDES, 2012a; NASCIMENTO JUNIOR et al., 2016; PAES et al., 2016).

Tratando-se das análises entre cada momento da competição (início e final), 
era esperado que os resultados do final da competição fossem melhores que os resultados do início da competição, pois, de acordo com a literatura (NASCIMENTO JUNIOR et al., 2016; PAES et al., 2016), era esperado que a experiência tivesse influência nos resultados do estudo. Dessa forma, era esperado que os resultados do final da competição demonstrassem escores de menores de ansiedade e escores maiores de autoconfiança, quando comparados com os resultados apresentados no início da competição. Além disso, como os atletas conquistaram inúmeras vitórias no decorrer da competição, alcançando a final do campeonato, era esperado que os atletas apresentassem maiores escores de autoconfiança na final em relação ao início da competição.

Porém, isso não ocorreu, pois que os resultados não demonstraram diferenças entre os resultados do início e do final da competição. Tais achados não corroboram os apresentados pela literatura (FERNANDES et al., 2014; NASCIMENTO JUNIOR et al., 2016; NOGUEIRA, 2016; PAES et al., 2016; VIEIRA et al., 2011). Entretanto, a literatura supracitada investigou atletas de elite, o que não foi o caso do presente estudo e, por isso, pode ter ocorrido divergência nos resultados.

De acordo com a literatura (FERNANDES et al., 2013) os atletas, quando mais experientes, identificam os sinais do ambiente e os interpretam como facilitadores para o desempenho, como por exemplo, os sinais de ansiedade e autoconfiança. Em contrapartida, os atletas, quando menos experientes, interpretam os sinais de ansiedade e autoconfiança como ruídos, o que dificulta o desempenho (FERNANDES et al., 2014). Entretanto, como já mencionado, no presente estudo esses resultados não foram encontrados, pois não houve diferença significantes entre os momentos de coleta.

Era esperado que no final da competição os atletas tivessem maior capacidade de identificar as informações do contexto e, com isso, apresentassem comportamentos diferentes aos encontrados no início da competição, mas isso não ocorreu. Uma possível explicação para isso pode ter relação com os participantes do presente estudo, já que os mesmos não são atletas de elite. De acordo com a literatura (PAES et al., 2016), os atletas amadores (atletas de base) apresentam níveis baixos de ansiedade somática e cognitiva, possivelmente pela menor cobrança que se tem sobre o atleta, comparado com o atleta de alto rendimento (BARRETO, 2017; BOAS et al., 2012; FERNANDES et al., 2014; PAES et al., 2016; SOUZA; TEIXEIRA; LOBATO, 2012).

Além disso, o papel do técnico parece ter influência nos níveis de ansiedade dos atletas. De acordo com a literatura (VIEIRA et al., 2013), quanto mais o treinador for efetivo e aproveitar as oportunidades de ensino, maior será o seu impacto sobre o desenvolvimento do atleta, o que pode ser entendido, no presente estudo, como influência nos níveis de ansiedade. Somado a isso, Gomes e Machado (2010) apontam que, embora existam imensas variáveis que condicionam e 
explicam a eficácia dos treinadores, as ações otimistas, motivacionais e favoráveis em relação as atividades impostas aos atletas estão associadas positivamente com os níveis de satisfação dos atletas e, assim, diminuem a sensação de cobrança (percepção de ações externas) do atleta frente ao contexto.

Tais informações reforçam a importância do técnico no processo de organização e construção de um grupo satisfeito e com motivação para prática esportiva, corroborando a literatura (MESQUITA et al., 2009; RIBEIRO, 2016). Cabe apontar que essas hipóteses explicativas não foram objetivo deste estudo, demonstrando perspectivas futuras de estudo e limitações do presente estudo. Dando continuidade, um fator que pode ter contribuído para a não superioridade dos valores de autoconfiança do final da temporada, diz respeito ao fato dos atletas terem sido avaliados na final do campeonato.

De acordo com Machado (2006), a final de um campeonato pode ser interpretada pelos atletas como algo ameaçador, pois, após a final, os atletas podem ser considerados "heróis" ou "vilões". Assim, o que pode ter ocorrido no presente estudo, é que os atletas, em função da situação (final do campeonato), encontraram-se apreensivos com a incerteza do resultado, o que não contribuiu para o aumento da autoconfiança (FERNANDES et al., 2013). Todavia, essa hipótese deveria conduzir os atletas a aumentarem os níveis de ansiedade, o que no presente estudo não ocorreu, refutando essa hipótese.

Talvez, o aumento dos níveis de ansiedade não ocorreu em função das intervenções da comissão técnica e da psicóloga que acompanha a equipe, o que demonstra, como supracitado, a importância da preparação do técnico e da comissão técnica no acompanhamento dos atletas no decorrer das competições (WEINBERG; GOULD, 2017). Tais apontamentos demonstram a importância do psicólogo do esporte na preparação dos atletas, principalmente frente aos atletas na formação, pois as cobranças externas pela vitória podem influenciar diretamente os níveis de ansiedade e, com isso, os atletas podem interpretar tais informações como dificultadoras para o desempenho (FERNANDES et al., 2013). Enquanto o papel da psicóloga, não se fez o acompanhamento das intervenções realizadas por ela, o que limita a extrapolação das discussões, demonstrando perspectivas futuras de estudo e limitações do presente estudo.

Diante dos resultados supracitados, pode-se citar que existe relevância na presença do psicólogo do esporte no acompanhamento dos atletas. Além disso, os psicólogos do esporte, ao prepararem sua periodização de treinamento psicológico, podem propor estratégias de aumento da autoconfiança (COWDEN; FULLER; ANSHEL, 2014), pois a mesma apresenta-se como um fator que diminuiu a ansiedade, o que é denominado pela literatura de tenacidade. Dessa forma, a literatura apresenta resultados de que as estratégias de autoconfiança podem funcionar como uma ferramenta de coping (NASCIMENTO JUNIOR et al., 2016), auxiliando os atletas no enfrentamento da ansiedade que a competição gera. 
Assim, no presente estudo, a autoconfiança pode ter atuado como estratégia de coping e, assim, contribuído para o baixo escore de ansiedade (cognitiva e somática), corroborando outros estudos com atletas (BERTUOL; VALENTINI, 2006; HAGAN; POLLMANN; SCHACK, 2017; SEELEY et al., 2000; TERTULIANO et al., 2019; VIEIRA et al., 2011).

Os resultados encontrados no presente estudo sugerem que os atletas foram capazes de lidar com fatores estressores da competição e não comprometeram seu rendimento na mesma (BANDURA, 1997), haja vista os mesmos terem participado das finais da competição disputada. Somado a isso, pode-se observar que a literatura aponta que atletas autoconfiantes apresentam-se mais à vontade nas competições e, consequentemente, concentram-se mais durante as partidas, o que facilita o desempenho (BERNARDT; SEHNEM, 2017; TSOPANI; DALLAS; SKORDILIS, 2011).

Diante disso, o presente estudo apresenta o avanço científico no que tange a importância da autoconfiança para o controle da ansiedade em atletas de categorias de base do basquetebol, corroborando a literatura que investigou adultos (BERNARDT; SEHNEM, 2017; CARRON et al., 2002; FORTES et al., 2017; LUNDQVIST; KENTTÄ; RAGLIN, 2011; MODROÑO; GUILLÉN, 2011; PAES et al., 2016; PONS et al., 2018; TERTULIANO et al., 2019).

Finalizando, o presente estudo apresenta as seguintes limitações: (a) ter investigado atletas de categorias de base; (b) de um único clube; (c) apenas do sexo masculino; (d) treinados pelo mesmo treinador; e (e) não ter acompanhado o trabalho da psicóloga junto aos atletas e comissão técnica. Além disso, o presente estudo apresenta a limitação de não ter relacionado as variáveis investigadas com outras variáveis, como coesão de grupo, resultados no campeonato $e$, estratégias adotadas na preparação psicológica o perfil do treinador. Em relação ao uso apenas da dimensão intensidade do CSAI-2r, esse fato não é considerado uma limitação haja vista o uso somente dessa dimensão ter suporte teórico (FERNANDES; VASCONCELOS-RAPOSO; FERNANDES, 2012a; TERTULIANO et al., 2019).

\section{CONSIDERAÇÕES FINAIS}

Os resultados do presente estudo demonstraram que atletas de basquetebol investigados, apresentaram, em ambos os momentos de coleta (início e final da competição), elevados níveis de autoconfiança e baixos níveis de ansiedade (somática e cognitiva). Além disso, os resultados não demonstraram mudanças significantes entre os dois momentos coletados. Esses resultados corroboram a teoria multidimensional da ansiedade (MARTENS; VEALEY; BURTON, 1990). Em síntese, os resultados demonstraram que os atletas investigados, ao apresentam 
elevados níveis de autoconfiança, apresentaram baixos níveis de ansiedade.

Assim, para estudos futuros, sugere-se que a amostra seja constituída por atletas de ambos os sexos, que se compare os resultados com diferentes modalidades esportivas, que se faça a relação dos níveis de ansiedade com outras variáveis, com, por exemplo, o perfil do treinador, que se acompanhe o trabalho do psicólogo do esporte junto aos atletas para traçar a relação entre os níveis de ansiedade e as estratégias de treinamento psicológico adotadas por ele, além de se conduzir pesquisas acerca da ansiedade utilizando-se da avaliação das demais dimensões (frequência e direção), como forma de auxiliar a compreensão da influência dos níveis de ansiedade no rendimento esportivo.

\section{REFERÊNCIAS}

ARRUDA, Ademir F. S.; AOKI, Marcelo S.; PALUDO, Ana Carolina; MOREIRA, Alexandre. Salivary steroid response and competitive anxiety in elite basketball players: Effect of opponent level. Physiology \& behavior, Zurich, v. 177, n. 1, p. 291-296, 2017. Disponível em: https://pubmed.ncbi.nlm.nih.gov/28527679/ .

BANDURA, Albert. Self-efficacy: The exercise of control. New York: Freeman, 1997.

BARRETO, Patrícia Marques. Perfil de estado de humor, ansiedade-traço e ansiedadeestado em jovens ginastas. 2017. 53 f. Dissertação (Mestrado em Psicologia do Desenvolvimento e Aprendizagem)- Universidade Estadual Paulista Júlio de Mesquita Filho, Bauru, 2017.

BERNARDT, Andréia Maria; SEHNEM, Scheila Beatriz. . Pesquisa em Psicologia: anais eletrônicos, Joaçaba, p. 37-44, 2017. Disponível em:

https://portalperiodicos.unoesc.edu.br/pp ae/article/view/15580.

BERTUOL, Lisandra; VALENTINI, Nadia Cristina. Ansiedade competitiva de adolescentes: Gênero, Maturação, Nível de experiência e Modalidades Esportivas. Revista da Educação Física da UEM, Maringá, v. 17, n. 1, p. 65-74, 2006. Disponível em:

http://periodicos.uem.br/ojs/index.php/RevEducFis/article/view/3378.

BIAGGIO, Angela M. B.; NATALÍCIO, Luiz; SPIELBERGER, Charles D. Desenvolvimento da forma experimental em português do Inventário de Ansiedade Traço-Estado (IDATE) de Spielberger. Arquivos Brasileiros de Psicologia Aplicada, Rio de Janeiro, v. 29, n. 3, p. 31-44, 1977. Disponível em:

http://bibliotecadigital.fgv.br/ojs/index.php/abpa/article/view/17827

BOAS, Marcelo Silva Villas; COELHO, Ricardo Weigert; VIEIRA, Lenamar Fiorese; FONSECA, Paulo Henrique Santos; KUCZYNSKI, Kátia Maria; BOAS, Angeli Graças B. Villas. Análise do nível de ansiedade de jovens atletas da Modalidade de Futsal. Conexões, Campinas, v. 10, n. 3, p. 77-86, 2012. Disponível em: https://periodicos.sbu.unicamp.br/ojs/index.php/conexoes/article/view/8637648.

CARRON, Albert V.; COLMAN, Michelle M.; WHEELER, Jennifer; STEVENS, Diane. Cohesion and Performance in Sport: A Meta Analysis. Journal of Sport and Exercise Psychology, Bath, v. 24, n. 2, p. 168-188, 2002. Disponível em: https://journals.humankinetics.com/view/journals/jsep/24/2/article-p168.xml. 
COELHO, Eduarda Maria; VASCONCELOS-RAPOSO, José; MAHL, Alvaro Cielo. Confirmatory factorial analysis of the Brazilian version of the Competitive State Anxiety Inventory-2 (CSAI-2). The Spanish journal of psychology, Madrid, v. 13, n. 1, p. 453-60, 2010. Disponível em: https://www.ncbi.nlm.nih.gov/pubmed/20480711.

COWDEN, Richard G.; FULLER, Dana K.; ANSHEL, Mark H. Psychological Predictors of Mental Toughness in Elite Tennis: An Exploratory Study in Learned Resourcefulness and Competitive Trait Anxiety. Perceptual and Motor Skills, Norfolk, v. 119, n. 3, p. 661-678, 2014. Disponível em: https://www.ncbi.nlm.nih.gov/pubmed/25387038.

COX, Richard H.; MARTENS, Matthew P.; RUSSELL, William D. Measuring Anxiety in Athletics: The Revised Competitive State Anxiety Inventory-2. Journal of Sport and Exercise Psychology, Bath, v. 25, n. 4, p. 519-533, 2003. Disponível em: https://journals.humankinetics.com/view/journals/jsep/25/4/article-p519.xml.

FERNANDES, Marcos Gimenes; NUNES, Sandra Adriana Neves; VASCONCELOS-RAPOSO, José; FERNANDES, Helder Miguel. Factors influencing competitive anxiety in Brazilian athletes. Brazilian Journal of Kinanthropometry and Human Performance, Florianópolis, v. 15, n. 6, p. 705-715, 2013. Disponível em:

https://periodicos.ufsc.br/index.php/rbcdh/article/view/1980-0037.2013v15n6p705.

FERNANDES, Marcos Gimenes; NUNES, Sandra Adriana Neves; VASCONCELOS-RAPOSO, José; FERNANDES, Helder Miguel. Efeitos da experiência nas dimensões de intensidade, direção e frequência da ansiedade e autoconfiança competitiva: Um estudo em atletas de desportos individuais e coletivos. Motricidade, Ribeira de Pena, v. 10, n. 2, p. 81-89, 2014. Disponível em:

http://www.scielo.mec.pt/scielo.php?script=sci arttext\&pid=S1646107X2014000200009.

FERNANDES, Marcos Gimenes; VASCONCELOS-RAPOSO, José; FERNANDES, Helder Miguel. Propriedades Psicométricas do CSAI-2 em Atletas Brasileiros. Psicologia: Reflexão e Crítica, Porto Alegre, v. 25, n. 4, p. 679-687, 2012a. Disponível em:

http://www.scielo.br/scielo.php?script=sci arttext\&pid=S0102-79722012000400007.

FERNANDES, Marcos Gimenes; VASCONCELOS-RAPOSO, José; FERNANDES, Helder Miguel. Relação entre orientações motivacionais, ansiedade e autoconfiança, e bem-estar subjetivo em atletas brasileiros. Motricidade, Ribeira de Pena, v. 8, n. 3, p. 4-18, 2012b. Disponível em: http://www.scielo.mec.pt/scielo.php?script=sci abstract\&pid=S1646$107 \times 2012000300002 \& \mathrm{lng}=\mathrm{pt \& n} r \mathrm{~m}=$ iso.

FIELD, Andy. Descobrindo a estatística usando o SPSS. São Paulo: Bookman, 2009.

FORTES, Leonardo S.; COSTA, Bruna D. V. da; PAES, Pedro P.; NASCIMENTO JÚNIOR, José Roberto A. do; FIORESE, Lenamar; FERREIRA, Maria E. C. Influence of CompetitiveAnxiety on Heart Rate Variability in Swimmers. Journal of Sports Science \& Medicine, Bursa Turkey, v. 16, n. 4, p. 498-504, 2017. Disponível em:

https://www.ncbi.nlm.nih.gov/pmc/articles/PMC5721179/.

GOMES, Antônio Rui; MACHADO, Afonso Antonio. Liderança, coesão e satisfação em equipas de voleibol portuguesas: Indicações da investigação e implicações práticas. In: BRANDÃO, Maria Regina Ferreira; MACHADO, Afonso Antonio. (Eds.). O Voleibol e a psicologia do esporte. São Paulo: Atheneu, 2010. p. 187-218.

GREEN, Samuel B.; SALKIND, Neil J.; AKEY, Theresa M. Using SPSS for windows: analyzing and understanding data. 2. ed. New Jersey: Prentice Hall, 2000. 
HAGAN, John E.; POLLMANN, Dietmar; SCHACK, Thomas. Interaction between gender and skill on competitive state anxiety using the time-to-event paradigm: What roles do intensity, direction, and frequency dimensions play? Frontiers in Psychology, Lausanne, v. 8, n. 692, p. 1-13, 2017. Disponível em:

https://www.ncbi.nlm.nih.gov/pmc/articles/PMC5430064/.

HOPKINS, Will G. A scale of magnitudes for effect statistics. 2002. Disponível em: http://www.sportsci.org/resource/stats/index.html. Acesso em: 12 set. 2017.

INTERDONATO, Giovanna Carla; OLIVEIRA, Arli Ramos; LUIZ JUNIOR, Clovis Correa; GREGUOL, Márcia. Análise da ansiedade de traço competitiva em jovens atletas. Conexões, Campinas, v. 8, n. 3, p. 1-9, 2010. Disponível em:

https://doi.org/10.20396/conex.v8i3.8637722.

LUNDQVIST, Carolina; KENTTÄ, Goran; RAGLIN, John S. Directional anxiety responses in elite and sub-elite young athletes: intensity of anxiety symptoms matters. Scandinavian journal of medicine \& science in sports, Stockholm, v. 21, n. 6, p. 853-862, 2011. Disponível em: https://pubmed.ncbi.nlm.nih.gov/22126716/.

MACHADO, Afonso Antonio. Psicologia do esporte: da educação física escolar ao esporte de alto nível. Rio de Janeiro: Guanabara Koogan, 2006.

MARTENS, Rainer; BURTON, Damon; VEALEY, Robin S.; BUMP, Linda A.; SMITH, Daniel E. Development and validation of the Competitive State Anxiety Inventory-2. In: MARTENS, Rainer; VEALEY, Robin S.; BURTON, Damon (Eds.). Competitive anxiety in sport. Champaign: Human Kinetics, 1990. p. 117-232.

MARTENS, Rainer; BURTON, Damon; RIVKIN, F.; SIMON, Julie. Reliability and validity of the Competitive State Anxiety Inventory (CSAI). In: NADEAU, Claude H.; HALLIWELL, Wayne C.; NEWELL, Karl M.; ROBERTS, Glyn C. (Eds.). Psychology of motor behavior and sport - 1979. Champaign: Human Kinetics Publishers, 1980. p. 91-99.

MARTENS, Rainer; VEALEY, Robin S.; BURTON, Damon. Competitive anxiety in sports. Champaign: Human Kinetics, 1990.

MESQUITA, Isabel; FARIAS, Cláudio; OLIVEIRA, Guilherme; PEREIRA, Felissima. A intervenção pedagógica sobre o conteúdo do treinador de futebol. Revista Brasileira de Educação Física e Esporte, São Paulo, v. 23, n. 1, p. 25-38, 2009. Disponível em: http://www.revistas.usp.br/rbefe/article/view/16708.

MILAVIĆ, Boris; JURKO, Damir; GRGANTOV, Zoran. Relations of competitive state anxiety and efficacy of young volleyball players. Collegium antropologicum, Zagreb, v. 37, n. 2, p. 83-92, 2013. Disponível em:

https://pubmed.ncbi.nlm.nih.gov/23914493/

MODROÑO, Cristián; GUILLÉN, Félix. Anxiety Characteristics of Competitive Windsurfers: Relationships with Age, Gender, and Performance Outcomes. Journal of Sport Behavior, Mobile, v. 34, n. 3, p. 281-294, 2011. Disponível em:

https://www.researchgate.net/publication/224863630 Anxiety Characteristics of Comp etitive Windsurfers Age Gender Performance Outcome.

NASCIMENTO JUNIOR, José Roberto Andrade do; BALBIM, Guilherme Moraes; VISSOCI, João Ricardo Nickening; MOREIRA, Caio Rosas; PASSOS, Patrícia Carolina Borsato; VIEIRA, Lenamar Fiorese. Análise das relações entre ansiedade estado e coesão de atletas de handebol. Revista Psicologia: Teoria e Prática, São Paulo, v. 18, n. 2, p. 89102, 2016. Disponível em:

http://pepsic.bvsalud.org/scielo.php?script=sci abstract\&pid=S1516- 


\section{$\underline{36872016000200007 \& \text { lng }=p t \& n r m=i s o .}$}

NOGUEIRA, Francine Caetano Andrade. Crença de auto eficácia e ansiedade em atletas de Voleibol. 2016. 115 f. Tese (doutorado em Psicologia) - Instituto de Ciências Humanas, Universidade Federal de Juiz de Fora, Juiz de Fora, 2016.

PAES, Mayara Juliana; MACHADO, Thais do Amaral; BERBETZ, Sabrina Rebecca; STEFANELLO, Joice Mara Facco. Frequência, intensidade e direção da ansiedade e sua relação com a coesão grupal em uma equipe de voleibol infanto-juvenil masculina. Revista Brasileira de Psicologia do Esporte, São Paulo, v. 6, n. 3, p. 46-56, 2016. Disponível em: https://portalrevistas.ucb.br/index.php/RBPE/article/view/7276.

PALUDO, Ana Carolina; NUNES, Sandra Adriana Neves; SIMÕES, Antônio Carlos; FERNANDES, Marcos Gimenes. Relação entre ansiedade competitiva, autoconfiança e desempenho esportivo: uma revisão ampla da literatura. Psicologia Argumento, Curitiba, v. 34, n. 85, p. 156-169, 2016. Disponível em:

https://periodicos.pucpr.br/index.php/psicologiaargumento/article/view/23315

PONS, Joan; VILADRICH, Carme; RAMIS, Yago; POLMAN, Remco. The Mediating Role of Coping between Competitive Anxiety and Sport Commitment in Adolescent Athletes. The Spanish journal of psychology, Madrid, v. 21, p. E7, 2018. Disponível em: https://pubmed.ncbi.nlm.nih.gov/29576037/.

RIBEIRO, Catarina Correia. Qualidade da relação treinador-atleta em contextos desportivos: Relações com fatores de grupo e diferenças em função do sexo. 2016. $31 \mathrm{f}$. Dissertação (Mestrado em Psicologia aplicada) - Universidade do Minho, Braga, 2016.

SEELEY, G.; STOREY, J.; WAGNER, D.; WALKER, C.; WATTS, K. Anxiety levels and gender differences in social volleyball players before and during competition. SportPsyc Unpublished, Sydney, v. 1, p. 1-5, 2000. Disponível em:

http://www.geocities.ws/CollegePark/5686/su99p5.htm.

SONOO, Christi Noriko; GOMES, Anderson Luiz; DAMASCENO, Mara Laiz; SILVA, Schelyne Ribas da; LIMANA, Mirieli Denardi. Ansiedade e desempenho: um estudo com uma equipe infantil de voleibol feminino. Motriz, Rio Claro, v. 16, n. 3, p. 629-637, 2010. Disponível em: https://www.scielo.br/scielo.php?pid=S1980-

$65742010000300010 \&$ script $=$ sci abstract\&tIng=pt.

SOUZA, Mário Alberto Pereira; TEIXEIRA, Robson Bonoto; LOBATO, Paulo Lanes. Manifestação da ansiedade pré-competitiva em nadadores amadores. Revista da Educação Física da UEM, Maringá, v. 23, n. 2, p. 195-203, 2012. Disponível em: http://www.scielo.br/scielo.php?script=sci arttext\&pid=S1983-30832012000200004.

SPIELBERGER, C. D. Anxiety: current trends in theory and research. 1. ed. New York: Academic Press, 1972.

SPIELBERGER, Charles D.; GORSUCH, R. L.; LUSHENE, R. E.; Manual for the State-Trait Anxiety Inventory. Palo Alto: Consulting Psychologists Press, 1970.

TEIXEIRA, Karen Cristine. Construção e busca de evidências de validade de precisão de uma medida de ansiedade para atletas. 2016. 139 f. Dissertação (Mestrado em Psicologia) - Centro de Filosofia e Ciências Humanas, Universidade Federal de Santa Catarina, Florianópolis, 2016.

TEIXEIRA, Karen Cristine; NUNES, Carlos Henrique Sancineto da Silva; CRUZ, Roberto Moraes. Estudos psicométricos de instrumentos de ansiedade no contexto esportivo: uma revisão sistemática. Revista Avaliação Psicológica, Itatiba, v. 15, n. 3, p. 362-370, 2016. 
Disponível em: http://pepsic.bvsalud.org/scielo.php?script=sci arttext\&pid=S167704712016000400010.

TERTULIANO, Ivan Wallan; BUZETO, Fernanda Karen; OLIVEIRA, Vivian de; MACHADO, Afonso Antonio; MONTIEL, José Maria. Os efeitos da idade e do sexo no nível de ansiedade de nadadores de águas abertas. Conexões: Educação Física, Esporte e Saúde, Campinas, v. 17, n. e019021, p. 1-19, 2019. Disponível em:

https://periodicos.sbu.unicamp.br/ojs/index.php/conexoes/article/view/8655148

TSOPANI, Despoina; DALLAS, George; SKORDILIS, Emmanouil K. Competitive state anxiety and performance in young female rhythmic gymnasts. Perceptual and motor skills, Norfolk, v. 112, n. 2, p. 549-560, 2011. Disponível em:

https://journals.sagepub.com/doi/abs/10.2466/05.09.20.PMS.112.2.549-560.

VIEIRA, Lenamar Fiorese; CARRUZO, Nayara Malheiros; AIZAVA, Paulo Vitor Suto;

RIGONI, Patrícia Aparecida Gaion. Análise da síndrome de "burnout" e das estratégias de "coping" em atletas brasileiros de vôlei de praia. Revista Brasileira de Educação Física e Esporte, São Paulo, v. 27, n. 2, p. 269-276, 2013. Disponível em:

https://www.scielo.br/scielo.php?script=sci arttext\&pid=S1807-55092013000200010.

VIEIRA, Lenamar Fiorese; TEIXEIRA, Cesar Luis; VIEIRA, José Luis Lopes; OLIVEIRA FILHO, Albertino. Autoeficácia e nível de ansiedade em atletas jovens do atletismo paranaense. Revista Brasileira de Cineantropometria e Desempenho Humano, Florianópolis, v. 13, n. 3, p. 183-188, 2011. Disponível em:

https://www.scielo.br/scielo.php?script=sci arttext\&pid=S1980-00372011000300004.

WEINBERG, Robert S.; GOULD, Daniel. Fundamentos da psicologia do esporte e do exercício. 6. ed. Porto Alegre: ArtMed, 2017. 